\title{
Analysis of Mutant Isocitrate Dehydrogenase 1 Immuno- expression, Ki-67 and Programmed Death Ligand 1 in Diffuse Astrocytic Tumours : Study of Single Center in Bandung, Indonesia
}

\author{
Hendrikus Masang Ban Bolly, ${ }^{1-3}$ Ahmad Faried, ${ }^{1,4}$ Yulius Hermanto, ${ }^{1,4}$ Billy Parulian Lubis, ${ }^{5}$ Firman Priguna Tjahjono, \\ Bethy Suryawathy Hernowo, ${ }^{5}$ Muhammad Zafrullah Arifin ${ }^{1}$ \\ Department of Neurosurgery, Faculty of Medicine, Universitas Padjadjaran-Dr. Hasan Sadikin Hospital, Bandung, Indonesia \\ Doctoral Program in Medicine, ${ }^{2}$ Faculty of Medicine, Universitas Padjadjaran, Bandung, Indonesia \\ Department of Biochemistry, ${ }^{3}$ Faculty of Medicine, Cenderawasih University, Jayapura, Indonesia \\ Oncology and Stem Cell Working Group, ${ }^{4}$ Faculty of Medicine, Universitas Padjadjaran, Bandung, Indonesia \\ Department of Anatomical Pathology, Faculty of Medicine, Universitas Padjadjaran-Dr. Hasan Sadikin Hospital, Bandung, Indonesia
}

Objective : Diffuse astrocytic tumour (DAT) is a diffuse infiltrative astrocytoma tumour accompanied by molecular parameters such as the presence or absence of isocitrate dehydrogenase (IDH) gene mutations. Ki-67 is a marker for DAT proliferation, while programmed death ligand 1 (PD-L1) indicates an immune evasion mechanism. This study aimed to analyze the correlation among mutant IDH1 R132H, Ki-67, and PD-L1 immunoexpression in the DAT.

Methods : A cross-sectional study was carried out on 30 paraffin blocks of DAT cases. Paraffin block samples consist of grade II $(n=14)$, grade III $(n=8)$, and grade IV ( $n=8)$. In this study, the immunohistochemistry-staining of mutant IDH1 R132H, Ki-67, and PD-L1 were carried out to determine the frequency of DAT with IDH1 mutations.

Results : Our study shown the frequency of IDH1 mutations in grade II 50.0\% (7/14), grade III 37.5\% (3/8), and grade IV $12.5 \%(1 / 8)$. Our study also showed a difference in Ki-67 and PD-L1 expression between each the degree of DAT histopathology ( $p=0.0001$ and $p=0.002$, respectively). There was an association between both mutant IDH1 R132H, and Ki-67 with PD-L1 expression in DAT ( $p=0.0087$ and $p=0.0049$, respectively).

Conclusion : DAT with the mutant IDH1 is frequently observed in grade II and small number of grade III. The expression of wild type IDH1, Ki-67, and PD-L1 were found to be higher in high grade DAT (grade III and grade IV). There is a correlation between each of mutant IDH1 status and Ki-67 with PD-L1 expression in DAT.

Key Words : Diffuse astrocytic tumor · Histopathology grade · Mutant IDH1 R132H · Ki-67 · PD-L1.

- Received : March 9, 2020 •Revised : April 6, 2020 •Accepted : May 12, 2020

- Address for reprints : Ahmad Faried

Department of Neurosurgery, Faculty of Medicine, Universitas Padjadjaran-Dr. Hasan Sadikin Hospital, Bandung, West Java 40161, Indonesia

Tel : +62-22-204-1694, Fax : +62-22-203-5836, E-mail : faried.fkup@gmail.com, ORCID : https://orcid.org/0000-0003-2967-8125

This is an Open Access article distributed under the terms of the Creative Commons Attribution Non-Commercial License (http://creativecommons.org/licenses/by-nc/4.0) which permits unrestricted non-commercial use, distribution, and reproduction in any medium, provided the original work is properly cited. 


\section{INTRODUCTION}

Glioma is the most common type of primary brain tumour which comes from glial cells. The types of glial tumours consist of astrocytomas, oligodendroglioma, or a mixture of both $^{18,26)}$. Based on World Health Organization (WHO) in 2016, the latest classification for astrocytoma tumours are diffuse astrocytic tumours (DATs). Definition of DAT is an accompanied infiltrative diffuse astrocytoma tumour with molecular parameters in the form of the presence or absence of isocitrate dehydrogenase (IDH) gene mutations ${ }^{14)}$. Of all intracranial tumours, the number of astrocytoma cases is around $40-43 \%$ with an annual average incidence of 5 per 100000 people and tend to recure ${ }^{7,18)}$. Based on GLOBOCAN International Agency for Research data on Cancer (IARC) in 2018, the incidence of brain tumours and nervous system ranks 16 th and the death due to this tumour ranks 13th in Indonesia $^{8)}$.

Brain tumour classification based on the latest WHO (2016), created and divided based on the growth patterns and behaviour of the tumour and its biomolecular examination, which is based on mutant or wild type of IDH status. Change the classification by entering genomic profile or molecular parameters especially in DAT, pose its own challenges in terms of the type of inspection and reporting the tumour ${ }^{14,15,20)}$. The foregoing becomes the basis in determining the entity classification of tumours of the central nervous system based on microscopic examination and molecular parameters seen from the genetic basis of the tumour genesis process ${ }^{14)}$.

Immunohistochemistry (ICH) markers, Ki-67, has been widely used as a marker of proliferation of tumour cells in humans. In recent studies, the molecular function of these protein markers has become easier to understand. Ki-67 has a role in the interphase and process of cell mitosis as well as changes in cellular distribution during cell cycle progression ${ }^{23)}$. In DAT, Ki-67 is used as an index of tumour cell proliferation in determining histopathological degrees aimed at predicting the biological behaviour of tumours and the prognosis for patients $^{25)}$. Ki-67 proliferation markers are cell nuclei antigens expressed in the Gl, S, G2, and M phases in the cell cycle and not expressed during the resting phase ${ }^{4)}$.

Recently, immunotherapy is one of the four pillars of cancer therapy in addition to surgical, radiation therapy, chemotherapy $^{10)}$. At the time of the initial immunotherapy study, more focused on acceleration of T-cells. Currently inhibitors of immuno-check points is the emerging research into the anti-tumour immune response. Successful clinical trial results with programmed death 1 (PD-1) antibodies and many other immune-checkpoint inhibitors, have opened new discoveries in field of cancer immunology ${ }^{10)}$. Target immune checkpoint from anti-cancer immunotherapy with the PD-1 antibody $(\mathrm{AB})$ and the ligand programmed death ligand 1 (PD-L1), is a receptor ligand trans membrane and $\mathrm{T}$-cell negative regulator signals that increase in some tumours including glioma and bind to PD-1 in T-cells, B-cells, dendritic cells and natural killer T cells, making tumour cells have a mechanism to be able to avoid attacks of immunity until they occur suppression of immune cells ${ }^{12,29)}$. In Indonesia, IDH mutation and immune-checkpoints have not been carried out routinely so that the data amount the percentage of DATs based on the molecular profile of IDH mutation status and PD-L1 has not been obtained.

\section{MATERIALS AND METHODS}

Ethical Approval was obtained from Committee of Ethics of Faculty of Medicine, Universitas Padjadjaran (approval No. 249/UN6.KEP/EC/2019). This retrospective study included 30 patients, who underwent 74 surgical resection or biopsy from January 2016 to December 2018, in which the diagnosis of primary gliomas was established. Clinical data were collected from patients histopathology and surgery reports. Surgically resected tumour tissues were fixated in 10\%-formaldehyde and preserved in paraffin-embedded blocks. Tumours then classified and graded according to 2016 WHO classification of central nervous system tumours ${ }^{13)}$. All patients received standard treatment for gliomas. The enrolled patients were followed-up to obtain the overall survival rate (median follow-up time, 30 months).

\section{IHC analysis}

Four micron thickness slides from the tumour tissue were deparaffinization and rehydrated. Antigen retrieval was carried out by decloaking chamber (DC2008INTL; Biocare Medical, Pacheco, CA, USA) at $100^{\circ}$ for 20 minutes using antigen retrieval solution (Tris EDTA $10 \mathrm{mmol} / \mathrm{L}, \mathrm{pH}$ 9.0). Sections then allowed for cooling at room temperature, followed by washing 
$2 \times$, each for 5 minutes, in phosphate buffered saline (PBS). Endogenous peroxidase activity was blocked by dipping sections in 3\% $\mathrm{H}_{2} \mathrm{O}_{2}$ blocker (Boster Biological Technology, Pleasanton CA, USA) for 10 minutes and washed in three changes of PBS. After the initial processing step, sections were incubated with primary antibodies anti-human glial fibrillary acidic protein (GFAP) AB (CP 040 Biocare, Clone N/A, rabbit monoclonal $\mathrm{AB}$, at 1 : 150 dilution); anti-human Ki-67 AB (Cellmarquee, Clone SP6, rabbit monoclonal AB, at 1 : 200 dilution); anti-human IDH1 R132H mutant specific AB (GTX57185 Genetex,
IHC 132, mouse monoclonal AB, at $1: 200$ dilution); anti-human PD-L1 AB (ab205921 Abcam, clone 28-8, rabbit monoclonal $\mathrm{AB}$, at $1: 200$ dilution) at the room temperature, respectively; followed by incubation with polyHRP non-biotin detection system for 30 minutes. Finally, the sections were counterstained with hematoxylin \& eosin, dehydrated and mounted. Positive results showed strong cytoplasmic and weak nuclear staining which appear 98 only in the tumour cells and the negative controls are prepared simultaneously for all 30 samples by replacing the primary $\mathrm{AB}$ with distilled water.

Table 1. Characteristics of the cohort

\begin{tabular}{|c|c|c|c|c|}
\hline & Grade II & Grade III & Grade IV & $p$-value \\
\hline Age & $36.71 \pm 15.52$ & $47.88 \pm 11.67$ & $54.38 \pm 11.76$ & $0.0468^{*}$ \\
\hline \multicolumn{5}{|l|}{ Sex } \\
\hline Male & 9 & 6 & 5 & 0.8402 \\
\hline Female & 5 & 2 & 3 & \\
\hline \multicolumn{5}{|l|}{ Chemotherapy } \\
\hline No & 14 & 7 & 0 & $<0.0001^{\dagger}$ \\
\hline Yes & 0 & 1 & 8 & \\
\hline \multicolumn{5}{|l|}{ Radiotherapy } \\
\hline No & 14 & 1 & 0 & $<0.0001^{\dagger}$ \\
\hline Yes & 0 & 7 & 8 & \\
\hline \multicolumn{5}{|l|}{ Lobus } \\
\hline Single & 11 & 1 & 4 & $0.0112^{\ddagger}$ \\
\hline Multiple & 3 & 7 & 4 & \\
\hline \multicolumn{5}{|l|}{ Resection } \\
\hline Partial/biopsy & 3 & 1 & 0 & \\
\hline Gross total resection & 10 & 3 & 4 & 0.1139 \\
\hline Total resection & 1 & 4 & 4 & \\
\hline GFAP & $14 / 14$ & $8 / 8$ & $8 / 8$ & $>0.9999$ \\
\hline IDH1 status & & & & 0.2137 \\
\hline Wild type & 7 & 5 & 7 & \\
\hline Mutant & 7 & 3 & 1 & \\
\hline Ki-67 & & & & $0.0001^{\S}$ \\
\hline$<10 \%$ & 13 & 5 & 0 & \\
\hline$\geq 10 \%$ & 1 & 3 & 8 & \\
\hline PD-L1 & & & & $0.002^{\|}$ \\
\hline $0-50 \%$ & 7 & 2 & 0 & \\
\hline $50-80 \%$ & 7 & 2 & 2 & \\
\hline$>80 \%$ & 0 & 4 & 6 & \\
\hline
\end{tabular}

${ }^{*} p<0.05$ (Kruskal-Wallis). ${ }^{\dagger} p<0.0001$ (chi-square). ${ }^{\ddagger} p<0.05$ (chi-square). ${ }^{\S} p<0.001$ (chi-square). ${ }^{\|} p<0.01$ (chi-square). GFAP : glial fibrillary acidic protein, $\mathrm{IDH}$ : isocitrate dehydrogenase, PD-L1 : programmed death ligand 1 
Expression of mutant IDH1 R132H, Ki-67, and PD-L1 were determined by semi-quantitative assessment of the proportion of the positively stained tumour cells. Cases with $\geq 10 \%$ stained cells were label as positive; cases with $<10 \%$ stained cells were label as negative (Ki-67 and mutant IDH1) ${ }^{2,30)}$. Immuno-reactive for PD-L1 was counted from the cells distribution as 0 (negative), $1(<20 \%), 2(20-50 \%), 3(50-80 \%)$, or 4 (>80\%). The IHC samples were reviewed by expert of neuropathologists who were blinded to the patients' clinical information. The average counts of the three regions were used for the final report. The average scores counted by the pathologists were recorded as the final result. When there was a large difference between observers, the score was re-evaluated to reach an agreement.

\section{Statistical analysis}

We used R 3.6.0 (Free Software Foundation, Boston, MA,
USA) and GraphPad Prism 7.03 (GraphPad Software, San Diego, CA, USA). The chi-square, Kruskal-Wallis (age), t-test, was used to assess the of associations significance of IDH1 with PD-L1 expression; Mann-Whitney U test was used to assess the of associations significance of Ki-67 with PD-L1. The log rank Mantel-Cox test was used to assess the difference of survival rate. The Cox proportional hazard regression model (univariate and multivariate analysis) was performed to determine the hazard ratio for each prognostic values. The KaplanMeier was used to generate the survival plots. When $p$ value $<0.05$ considered as statistically significant.

\section{RESULTS}

\section{Characteristics of the cohort}

We enrolled 30 patients diagnosed as WHO criteria of astro-
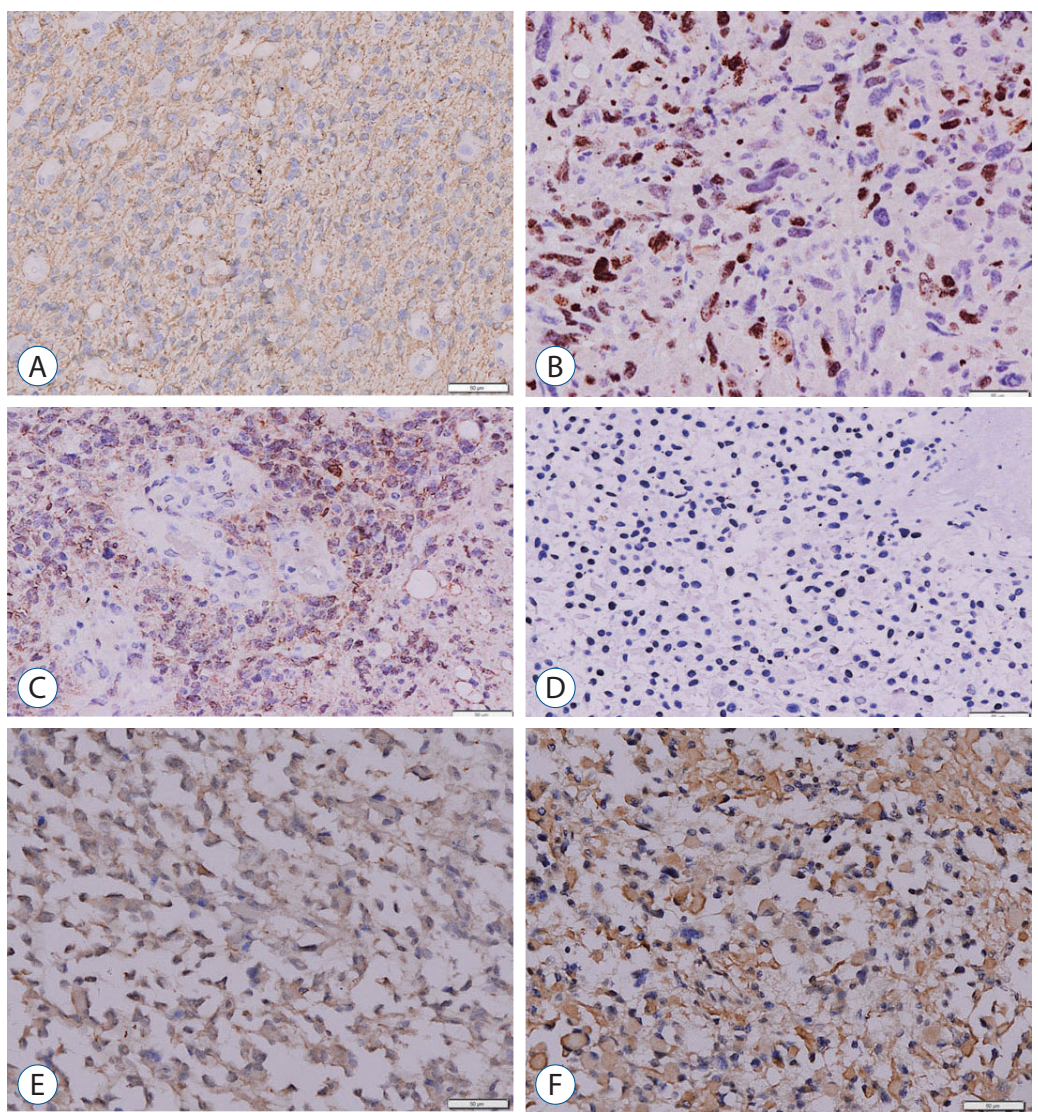

Fig. 1. Immunohistochemical detection of gliomas cells profile. Representative image of a glioma specimen that all cells positively stained with GFAP (A); high proliferation gliomas cells stained with $\mathrm{Ki}-67$ (B), mutant IDH1 R132H positive (C) and negative (D); PD-L1 positive $50-80 \%$ (E) and positive $>80 \%$ (F) are shown. Original magnification : ×100; scale bar : $50 \mu \mathrm{m}$. GFAP : glial fibrillary acidic protein, IDH : isocitrate dehydrogenase, PD-L1 : programmed death ligand 1. 
cytic gliomas $^{15)}$, 14 specimens were diagnosed as grade II, eight as grade III, and eight as grade IV (glioblastoma). Grade II was classified as low grade glioma (LGG), while grade III and IV were classified as high grade glioma (HGG). In this cohort, grade II patients were younger age $(36.71 \pm 15.52$ years $)$ than grade III (47.88 \pm 11.67 years) and grade IV patients $(54.38 \pm 11.76$, $p=0.0468$ ). The proportion of male patients was similar among grade II $(64.3 \%$; 9/14), grade III $(75 \% ; 6 / 8)$, and grade IV $(62.5 \% ; 5 / 8, p=0.8402)$. The higher level of astrocytic gliomas (grade III vs. IV) obtained not only radiotherapy $(p<0.0001)$ but must combined with chemotherapy $(p<0.0001)$. Grade III $(87.5 \% ; 7 / 8)$ and grade IV $(50 \% ; 4 / 4)$ were likely to have multiple-lobus involvement than grade II $(21.4 \% ; 3 / 14, p=0.0112)$. However, more grade III and IV patients obtained total and gross total resection $(93.75 \% ; 15 / 16)$ than grade II patients

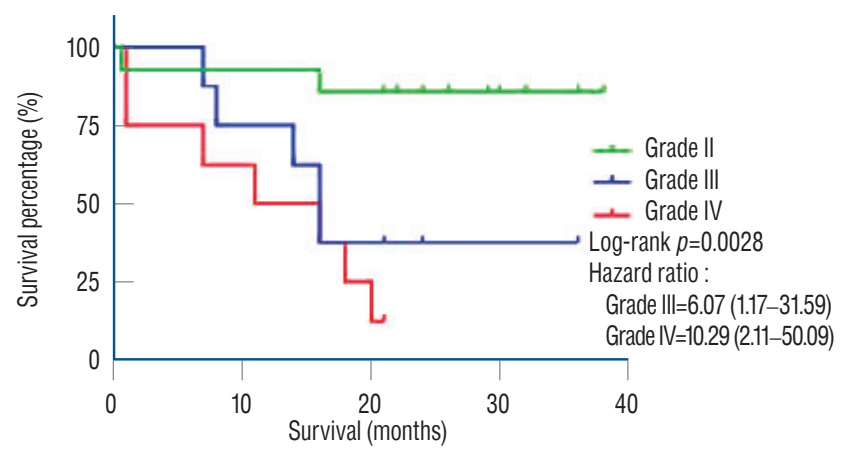

Fig. 2. Kaplan Meier Survival Plot of gliomas (World Health Organization grade II, III, and IV) patients; high grade glioma patients had a shorter 16 months survival rate vs. low grade glioma patients $(37.50 \% \pm 17.10 \%$ vs. $85.71 \% \pm 9.35 \%, p=0.0028$; hazard ratio [HR] grade III, 6.07 [1.17-31.59] and HR grade IV, 10.29 [2.11-50.09]). The patients in this cohort were followed-up after the diagnosis to gather the information regarding overall survival rate with the median of follow-up duration 30 months.
(78.6\%; 11/14, $p=0.1139)$. Patient characteristics are summarized in Table 1 .

\section{Pathological characteristic and $\mathrm{ICH}$ results of gliomas patients}

To gain some mechanistic knowledge that underlying malignancy in glioma patients, we performed IHC to examine several markers : GFAP, mutant IDH1 R132H, Ki-67, and PDL1. We confirmed that all tumour tissues in this cohort were astrocytic gliomas (100\% expression of GFAP, Fig. 1A). HGG tumour specimens were more proliferative (Ki-67 $\geq 10 \%$, grade III $37.5 \% ; 3 / 8$ and grade IV $100 \% ; 8 / 8)$ than LGG (7.14\%, 1/14, $p=0.0001$, Fig. 1B). Mutant IDH1 status, as one of the recommended examination by WHO, was often observed in LGG specimens $(50 \%$; 7/14) than HGG (grade III 37.5\%; 3/8 and grade IV $12.5 \% ; 1 / 8, p=0.2137$ respectively shown in Fig. $1 \mathrm{C}$ and D) patients. PD-L1 expression (50-80\% and $>80 \%$ PD-L1+ cells, Fig. 1E and F) was often observed in HGG specimens (grade III 75\%; 6/8 and grade IV 100\%; 8/8, respectively) than LGG $(50 \% ; 7 / 14, p=0.002)$. IHC results were summarized in Table 1.

\section{Survival probability of the cohort}

In this study, we found that the overall median survival of glioma patients was 18 months. To evaluate the underlying conditions, we analyzed the survival rate based on WHO grade, HGG patients had lower survival probability rate than LGG patients $(37.50 \% \pm 17.10 \%$ vs. $85.71 \% \pm 9.35 \%, p=0.0028$; hazard ratio [HR] grade III, 6.07 [1.17-31.59]; HR grade IV, 10.29 [2.11-50.09]; Fig. 2). Mutant IDH1 status had no correlation with Ki-67 ( $p=0.0634)$. The mutant IDH1 status was sig-
Association between Ki-67 and IDH1 status

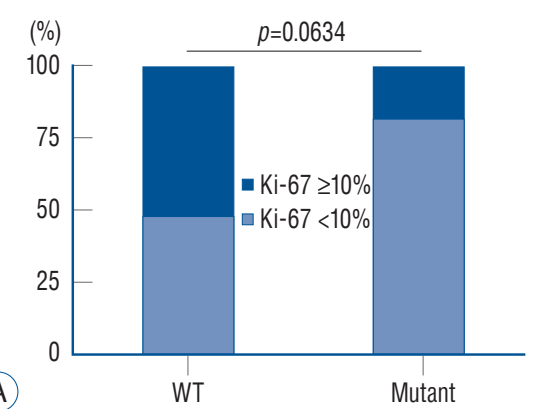

Association between IDH1 status and PD-L1 expression

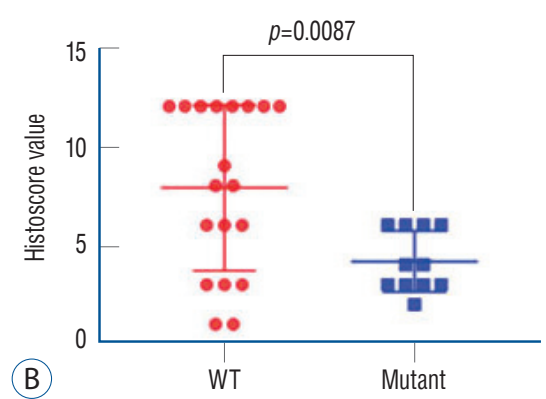

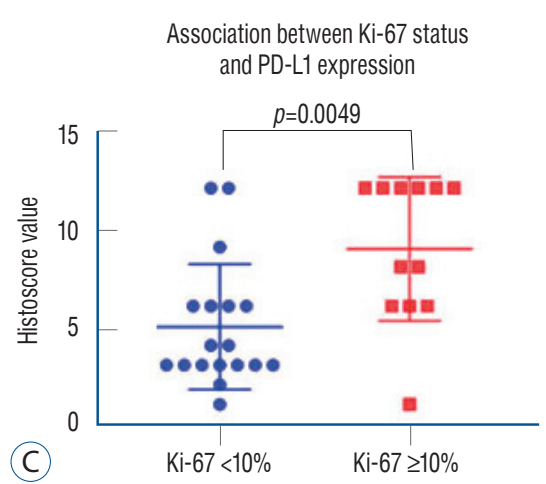

Fig. 3. Association between IDH1 status expression and Ki-67 or PD-L1. A : Association between Ki-67 and mutant IDH1. B : Association between IDH1 status and PD-L1 expression. C : Association between Ki-67 and PD-L1 expression. IDH : isocitrate dehydrogenase, PD-L1 : programmed death ligand 1. 
nificant inversely correlated with the higher PD-L1 immunopositivity ( $p=0.0087)$. The PD-L1 immuno-positivity correlated with Ki-67 ( $p=0.0049)$, the higher Ki-67 was associated with the higher PD-L1 expression (Fig. 3).

As shown in Table 2, by univariate analysis, grade IV patients had lower survival probability rate vs. grade II and III patients ( $p=0.0039$; HR, 10.29 [2.11-50.09]); patient with multiple lesions had lower survival probability rate vs. patients with single lesion only ( $p=0.0325$; HR, 0.28 [0.09-0.90]); Ki-67 $\geq 10 \%$ patients had lower survival probability rate vs. Ki-67 $<10 \%$ patients $(p=0.0016$; HR, 6.63 [2.05-21.40]); wild type
IDH1 patients had a tendency lower survival probability raye vs. mutant IDH1 patients, even not statistically significant $\left(p=0.9980\right.$; HR, $6.10 \times 10^{-9} 161$ [0-inf] $) ; \mathrm{PD}-\mathrm{L} 1>80 \%$ and $50-$ $80 \%$ patients had lower survival probability rate vs. PD-L1 $0-50 \%(p=0.0008$; HR, 7.46 [2.28-24.38]). By multivariate analysis, male patients had lower survival probability rate vs. female patients $(p=0.0222$; HR, $8.93[1.37-58.32])$ and Ki-67 $\geq 10 \%$ patients had lower survival probability rate vs. Ki-67 $<10 \%$ patients $(p=0.0211$; HR, $0.08[0.01-0.69])$.

Table 2. Cox proportional HR for clinical characteristics and immunostaining

\begin{tabular}{|c|c|c|c|c|c|}
\hline \multirow{2}{*}{ Factor } & \multirow{2}{*}{$\begin{array}{c}18 \text { months } \\
\text { survival rate (\%) }\end{array}$} & \multicolumn{2}{|c|}{ Univariate analysis } & \multicolumn{2}{|c|}{ Multivariate analysis } \\
\hline & & HR $(95 \% \mathrm{Cl})$ & $p$-value & HR (95\% CI) & $p$-value \\
\hline \multicolumn{6}{|l|}{ Age } \\
\hline Young ( $\leq 40$ years) & 75.00 & $0.64(0.20-2.03)$ & 0.446 & $1.01(0.008-0.08)$ & 0.9294 \\
\hline Old (>40 years) & 55.56 & Reference & & Reference & \\
\hline \multicolumn{6}{|l|}{ Sex } \\
\hline Male & 50.00 & $2.41(0.68-8.77)$ & 0.171 & $8.93(1.37-58.32)$ & $0.0222^{*}$ \\
\hline Female & 80.00 & Reference & & Reference & \\
\hline \multicolumn{6}{|l|}{ WHO grade } \\
\hline Grade 2 & 87.14 & Reference & & Reference & \\
\hline Grade 3 & 37.50 & $6.07(1.17-31.59)$ & 0.0321 & $7.71(0.68-87.12)$ & 0.0987 \\
\hline Grade 4 & 37.50 & $10.29(2.11-50.09)$ & $0.0039^{\dagger}$ & $0.87(0.04-19.27)$ & 0.9306 \\
\hline \multicolumn{6}{|l|}{ Lobus } \\
\hline Single & 81.25 & $0.28(0.09-0.90)$ & $0.0325^{*}$ & $4.39(0.45-42.45)$ & 0.2009 \\
\hline Multiple & 28.57 & Reference & & Reference & \\
\hline \multicolumn{6}{|l|}{ Surgery } \\
\hline Biopsi & 100.00 & Reference & & Reference & \\
\hline Subtotal & 52.94 & $2.19 \times 10^{9}(0-$ inf $)$ & 0.998 & $2.22 \times 10^{8}$ (0-inf) & 0.9991 \\
\hline Total & 33.33 & $3.13 \times 10^{9}$ (0-inf) & 0.998 & $3.39 \times 10^{8}$ (0-inf) & 0.9990 \\
\hline \multicolumn{6}{|l|}{ IDH1 status } \\
\hline Wild type & 36.84 & $1.64 \times 10^{9}(0-$ inf $)$ & 0.998 & $3.55 \times 10^{9}$ (0-inf) & 0.9985 \\
\hline Mutant & 100.00 & Reference & & Reference & \\
\hline \multicolumn{6}{|l|}{$\mathrm{Ki}-67$} \\
\hline$<10 \%$ & 77.78 & $0.15(0.05-0.49)$ & $0.0016^{\dagger}$ & $0.08(0.01-0.69)$ & $0.0211^{*}$ \\
\hline$\geq 10 \%$ & 33.33 & Reference & & Reference & \\
\hline \multicolumn{6}{|l|}{ PD-L1 } \\
\hline Low & 78.95 & $0.13(0.04-0.44)$ & $0.0008^{\ddagger}$ & $3.45(0.52-23.60)$ & 0.1993 \\
\hline High & 27.27 & Reference & & Reference & \\
\hline
\end{tabular}

${ }^{*} p<0.05$ (cox proportional hazard). ${ }^{\dagger} p<0.01$ (cox proportional hazard). ${ }^{\ddagger} p<0.001$ (cox proportional hazard). HR : hazard ratio, Cl : confidence interval, GFAP : glial fibrillary acidic protein, IDH : isocitrate dehydrogenase, PD-L1 : programmed death ligand 1 


\section{DISCUSSION}

Many genetic parameters recommended in the WHO 2016 classification, but somehow in many center like ours, may not have the ability to carry out molecular analyses. Studies shown that EGFR amplification and the combination of a positive finding for any two of the three other markers (pTERT mutant, or the chromosome 7/10 signatures) are highly specific for IDH WT GBM, while the combination of all three markers is exclusively seen in IDH WT GBM ${ }^{22}$. In our center, since the medical insurance system has not kept up with the pace of technical development and the limited national insurance budget, we could not freely apply IHC; this leads us try to analyze the clinical characteristic of our patients with results of limited IHC examination based on the WHO 2016 classification (status of IDH1); adding with common marker for proliferation (Ki-67) and immune-checkpoint (PD-L1).

This is the first study ever conducted in Bandung, Indonesia; the anti-IDH1 R132H immuno-expression was seen in 30 surgical or biopsy which had previously been diagnosed with WHO based diagnosis for glioma tumours. A total of 11 of the 30 samples analyzed turned out to have heterozygous mutations in the 132 IDH1 codon that specifically changed from arginine to histidine; Omer et al. ${ }^{17}$, shown a variation in the percentage of mutant IDH1 R132H for gliomas from several countries in Asia such as Japan reported 29.2-31.2\%; China 55.2-57.5\%; Korea 53.7\%; India 41.9\%; Iraq 34.86$37.1 \%$. Dwianingsih et al. ${ }^{5}$ in 2017, shown that IDH1 mutations are highly identified in Indonesian gliomas patients, as high as $74 \%(37 / 50)$ and significantly correlated with grading. However, in this study, we observed $36.7 \%$ (11/25) patients that possess IDH1 R132H mutation and exhibits no significant correlation with grading ( $p>0.05)$, it's in alignment with what has been observed by others ${ }^{21,24,27)}$; that stated the IDH1 mutation plays role in tumourigenesis of $\mathrm{LGG}^{27)}$.

Our patients average age of $36.71 \pm 15.52,47.88 \pm 11.67$, and $54.38 \pm 11.76$ in grade II, III, and IV, respectively ( $p=0.0468$ ). Other studies shown the median age of patients with and without IDH1 mutations are age 33 years and 53 years ${ }^{24}$. Our study in agreement with other that was specifically stated in glioblastoma, IDH1 mutations were significantly found in young age (mean, 47.9 years) and without IDH1 mutations in older age (mean, 60.6 years) ${ }^{24}$; in our study was 34,44 , and 55.5 years, respectively. In this cohort, patients with the higher grade treated with combination of chemo- and radio-therapy. Patients with the higher grade were mostly have multiple-lobus involvement in the brain than the lower grade.

Study results prove the frequency of dominant IDH1 mutations found in secondary glioblastoma compared to primary on $\mathrm{e}^{13,16}$. The predominant frequency of IDH1 mutations found predominantly in diffuse (grade II) astrocytoma tumours and anaplastic (grade III) astrocytomas has a tendency to develop into secondary glioblastoma. A negative IDH1 $\mathrm{R} 132 \mathrm{H}$ result (87.5\%) in glioblastoma does not necessarily prove that the sample tested is the primary glioblastoma; for reasons not checked for wild type IDH markers and there is still a possibility of mutations other than $\mathrm{R} 132 \mathrm{H}$ which may also occur in glioblastoma samples. This study specifically stated that there was no significant difference $(p=0.2137$, Table 1$)$ of the diagnostic ability of mutant IDH1 R132H in all three grade gliomas; also in this cohort, Ki-67 $\geq 10 \%$ had no correlation with IDH1 status ( $p=0.0634$ ), but the tendency Ki- $67 \geq 10 \%$ was associated with wild type IDH1 (Fig. 3A).

Recently found that wild type IDH is associated with significantly higher tumour infiltrating lymphocytes (TIL) and PD-L1 expression in all degrees of gliomas. In LGG and glioblastoma, PD-L1 expression was found to be higher in wild type IDH compared to mutant IDH ${ }^{11)}$. In wild type IDH glioma showed increased PD-L1 expression and TIL infiltration so that it was likely to be more immunologically active and more suitable for PD-1 immunomudolator therapy PD-L1 compared to mutant IDH. This is what underlies the importance of evaluating the molecular status of IDH1/2 in immunotherapy ${ }^{11}$. Our results show that in wild type IDH and Ki- $67 \geq 10 \%$ increasing the expression of PD-L1 (Fig. 3B and C; $p=0.0087$ and $p=0.0049$, respectively). There is a strong association between HGG and high Ki-67 expression and wild type IDH suggesting that HGG tumour cells were highly proliferative and metabolically active, hence triggering the expression of PD$\mathrm{L1}^{3,19)}$.

Wang et al. ${ }^{28)}$, who found that the PD-L1 expression in gliomas with mutants IDH1 showed low expression in different degrees of gliomas although statistically did not give significance in some groups. This indicates that the checkpoint immune response of PD-L1 is associated with wild type IDH1 in gliomas, which show different biological patterns between the two ${ }^{28}$. Berghoff et al. ${ }^{1)}$, found the result that in mutants IDH1 in gliomas with co-deletion 1p/19q showed the 
lowest PD-L1 expression results, followed by mutant IDH1 with non co-deletion $1 \mathrm{p} / 19 \mathrm{q}$, wild type IDH1 and glioblastoma which then gave increasing PD-L1 expression in their sequence. The PD-L1 expression statistically gave vary high significance to wild type IDH1 in grade II/III glioma compared to mutant IDH in grade II/III glioma. In this Berghoff study, no statistical difference was found between the level of PD-L1 expression in grade II/III glioma and glioblastoma with wild type IDH status ${ }^{11}$.

According to WHO 2016, diffuse gliomas are lumped together, regardless of their histopathological aspect (whether astrocytoma or oligodendroglioma); co-deletion $1 \mathrm{p} / 19 \mathrm{q}$ is a pathognomonic biomarker that defines a distinct gliomas entity; assessment of co-deletion $1 \mathrm{p} / 19 \mathrm{q}$, together with $I D H$ mutation and other markers (e.g., ATRX and TP53), can help distinguish oligodendrogliomas which are IDH-mutant status and co-deletion $1 \mathrm{p} / 19 \mathrm{q}$, from tumours of astrocytic lineage which are non co-deletion $1 \mathrm{p} / 19 \mathrm{q}^{15)}$. Since our limited budget grant, as mentioned above, to distinct our glioma-patient entity, we only performed GFAP IHC; as described Haah et al. ${ }^{9)}$, that GFAP is specific for astrocyte that can identified astrocytic component in gliomas. We confirmed that all tumour tissues in this cohort were astrocytic gliomas, as shown $100 \%$ moderate to strong expression of GFAP.

The mechanism of the relationship between IDH1 mutations and the micro-tumour environment still needs to be re-examined. The lower PD-L1 expression is associated with increased methylation promoter in gliomas with mutants IDH. The presence of a very high PD-L1 methylation promoter gene in mutants IDH compared to wild type IDH, indicates that the PD-L1 methylation promoter gene is likely to be associated with lower PD-L1 expression in mutant IDH vs. wild type IDH. The increase in methylation promoter is related to the characteristics of the hypermethylate phenotype in gliomas with mutants IDH that have been shown to be induced on 2-hydroxyglutarate oncometabolite. Other factors that influence differences in immune phenotypes between gliomas with mutant IDH and wild type IDH are epigenetic changes in the immune signaling pathway and the effect of 2-hydroxyglutarate on the micro-tumour environment including TIL $^{1)}$.

In order to see the bigger picture of our study, we try to validate our result by using Chinese Glioma Genome Atlas (CGGA) (http://www.cgga.org.cn) database, as for detail methodology described in our previous study ${ }^{6}$. In this study, we identified as follow : 1) there is an agreement between CGGA database (from total 1013 patients) with our results regarding correlation among IDH1 status, Ki-67 and PD-L1 (Fig. 4A and B); there is a tendency for wild type IDH1 gliomas to have a higher Ki-67 and PD-L1 expression, that indicating more malignant phenotype. 2) There is a statistically significant association between Ki-67 and PD-L1 ( $\mathrm{r}=0.096$, $p=0.0022$ ) (Fig. 4C). However, due to our small samples and for some of clinical factors (such as patient's age) is not-equal, a multi-center or nation-wide clinical studies in mandatory to overcome this limitations.

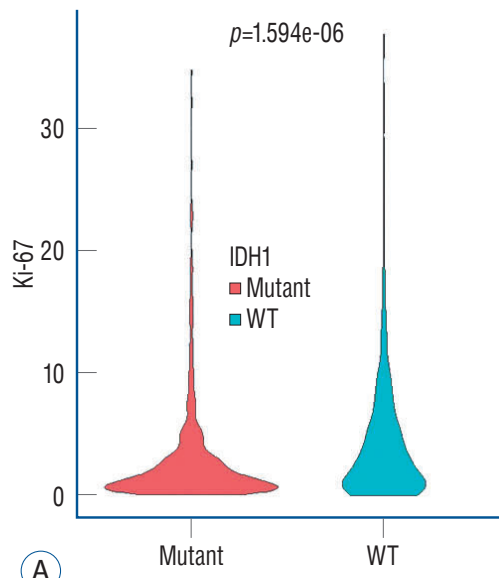

(A)
(B)

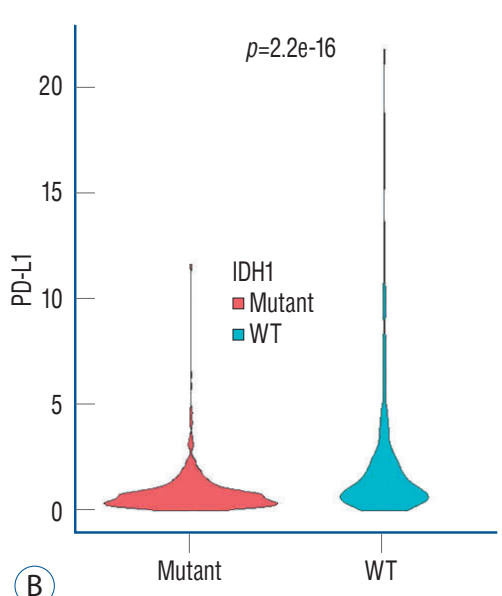

Fig. 4. Validation process using Chinese Glioma Genome Atlas database. Association between (A) Ki-67 and (B) PD-L1 with IDH1 status. C : Association between Ki-67 and PD-L1 expression. IDH : isocitrate dehydrogenase, PD-L1 : programmed death ligand 1.

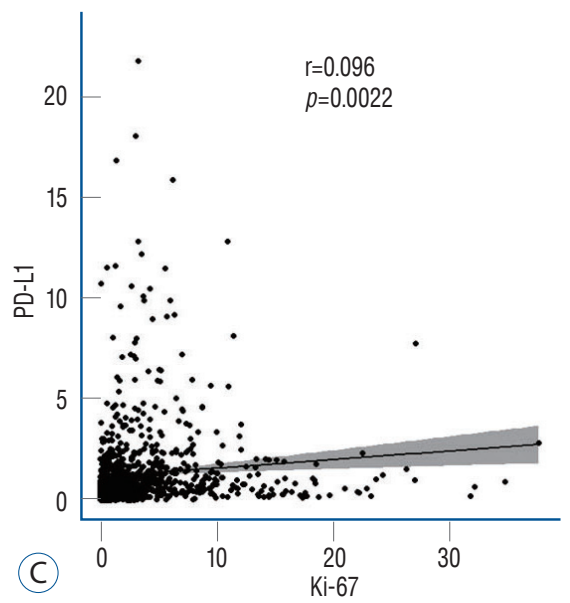




\section{CONCLUSION}

The frequency of mutant IDH1 R132H in grade II DAT in this study was lower than data from WHO. DAT with the mutant IDH1 is frequently observed in grade II and small number of grade III DAT. The expression of wild type IDH1, PDL1, and Ki-67 were found to be higher in DAT with high grade (grade III and IV). There is correlation between each of IDH1 R132H status with Ki-67 and PD-L1 expression in DAT. PDL1 examination can be performed for higher degree of DAT, to provide evidence for anti-PD-L1 target therapy.

\section{CONFLICTS OF INTEREST}

No potential conflict of interest relevant to this article was reported.

\section{INFORMED CONSENT}

Informed consent was obtained from all individual participants included in this study.

\section{AUTHOR CONTRIBUTIONS}

\author{
Conceptualization : AF, HMBB \\ Data curation : HMBBB, BPL \\ Formal analysis : $\mathrm{HMBB}, \mathrm{BSH}, \mathrm{YH}$ \\ Funding acquisition : AF, HMBB \\ Methodology : AF, BPL, BSH \\ Project administration : AF, FPT, MZA \\ Visualization : $\mathrm{YH}$ \\ Writing - original draft : AF \\ Writing - review \& editing : AF, YH, FPT, MZA
}

\section{ORCID}

Hendrikus Masang Ban Bolly https://orcid.org/0000-0002-8924-4495

Ahmad Faried https://orcid.org/0000-0003-2967-8125

Yulius Hermanto https://orcid.org/0000-0003-2937-8130

Billy Parulian Lubis ～https://orcid.org/0000-0002-6156-0306
Firman Priguna Tjahjono https://orcid.org/0000-0001-5827-8679

Bethy Suryawathy Hernowo https://orcid.org/0000-0003-2426-2534

Muhammad Zafrullah Arifin https://orcid.org/0000-0003-2529-9371

\section{- Acknowledgements}

Faried A. supported by the Grants-in-Aid from Indonesian Ministry of Research and Technology (National Research \& Innovation Agency) 16/E1/KPT/2020 for basic research.

\section{References}

1. Berghoff AS, Kiesel B, Widhalm G, Wilhelm D, Rajky O, Kurscheid S, et al. : Correlation of immune phenotype with IDH mutation in diffuse glioma. Neuro Oncol 19 : 1460-1468, 2017

2. Camelo-Piragua $S$, Jansen $M$, Ganguly $A$, Kim JC, Louis DN, Nutt CL : Mutant IDH1-specific immunohistochemistry distinguishes diffuse astrocytoma from astrocytosis. Acta Neuropathol 119 : 509-511, 2010

3. Chen RQ, Liu F, Qiu XY, Chen XQ : The prognostic and therapeutic value of PD-L1 in glioma. Front Pharmacol 9 : 1503, 2019

4. Das B, Raj KV, Atla B : Clinicohistopathological study of astrocytomas along with Ki-67 proliferative index. Int J Res Med Sci 6 : 665-670, 2018

5. Dwianingsih EK, Bawono RG, Rhamadianti AF, Bayuangga HF, Malueka RG : Correlation between grading and IDH1 mutation in Indonesian glioma. J Neurol Sci 381 : 386-387, 2017

6. Faried A, Hermanto Y, Tjahjono FP, Valentino A, Arifin MZ : Identification of periostin as a potential biomarker in gliomas by database mining. World Neurosurg 135 : e137-e163, 2020

7. Fu J, Shu J, Yu X, Pan J, Lv G, Zhou S, et al. : Predicting tumor recurrence of astrocytoma by $\mathrm{Ki}-67$ and proton magnetic resonance spectra. Int J Clin Exp Med 10 : 11187-11196, 2017

8. Global Cancer Observatory : International Agency for Research on Cancer: Indonesia. Available at : https://gco.iarc.fr/today/data/ factsheets/populations/360-indonesia-fact-sheets.pdf

9. Haah LE, Chong YG, Chu JW, Lee KC, Chae YS : Immunohistochemical study of gliomas using glial fibrillary acidic protein (GFAP), S-100 protein and neurofilament. J Korean Neurosurg Soc 20 : 389-398, 1991

10. Iwai Y, Hamanishi J, Chamoto K, Honjo T : Cancer immunotherapies targeting the PD-1 signaling pathway. J Biomed Sci 24 : 26, 2017

11. Kaminska B, Czapski B, Guzik R, Król SK, Gielniewski B : Consequences of IDH1/2 mutations in gliomas and an assessment of inhibitors targeting mutated IDH proteins. Molecules 24 : 968, 2019

12. Liu Y, Carlsson R, Ambjørn M, Hasan M, Badn W, Darabi A, et al. : PDL1 expression by neurons nearby tumors indicates better prognosis in glioblastoma patients. J Neurosci 33 : 14231-14245, 2013

13. Loenarz C, Schofield CJ : Expanding chemical biology of 2-oxoglutarate oxygenases. Nat Chem Biol 4 : 152-156, 2008 
14. Louis DN, Ohgaki H, Wiestler OD, Cavenee WK : WHO Classification of Tumours of the Central Nervous System. 4th ed. Vol. 1. France : International Agency for Research on Cancer, 2016, pp15-76

15. Louis DN, Perry A, Reifenberger G, von Deimling A, Figarella-Branger D, Cavenee WK, et al. : The 2016 World Health Organization classification of tumors of the central nervous system: a summary. Acta Neuropathol $131: 803-820,2016$

16. Nobusawa $S$, Watanabe $T$, Kleihues $P$, Ohgaki H : IDH1 mutations as molecular signature and predictive factor of secondary glioblastomas. Clin Cancer Res 15 : 6002-6007, 2009

17. Omer NS, Jalal JA, Ismael AT : IDH1 (R132H) imunoexpression in glioma. J Kurdistan Board of Med Specialities 4 : 57-63, 2018

18. Ostrom QT, Bauchet L, Davis FG, Deltour I, Fisher JL, Langer $C E$, et al. : The epidemiology of glioma in adults: a "state of the science" review. Neuro Oncol 16 : 896-913, 2014

19. Parsa AT, Waldron JS, Panner A, Crane CA, Parney IF, Barry JJ, et al. : Loss of tumor suppressor PTEN function increases B7-H1 expression and immunoresistance in glioma. Nat Med $13: 84-88,2007$

20. Röver LK, Gevensleben H, Dietrich J, Bootz F, Landsberg J, Goltz D, et al. : PD-1 (PDCD1) promoter methylation is a prognostic factor in patients with diffuse lower-grade gliomas harboring isocitrate dehydrogenase (IDH) Mutations. EBioMedicine 28 : 97-104, 2018

21. Saeed MS : IDH1 mutation in gliomas in Mosul city - Iraq. Open Access Maced J Med Sci 3 : 250-255, 2015

22. Stichel D, Ebrahimi A, Reuss D, Schrimpf D, Ono T, Shirahata M, et al. : Distribution of EGFR amplification, combined chromosome 7 gain and chromosome 10 loss, and TERT promoter mutation in brain tumors and their potential for the reclassification of IDHwt astrocytoma to glioblastoma. Acta Neuropathol $136:$ 793-803, 2018

23. Sun $X$, Kaufman PD : Ki-67: more than a proliferation marker. Chromosoma $127: 175-186,2018$

24. Thota B, Shukla SK, Srividya MR, Shwetha SD, Arivazhagan A, Thennarasu $\mathrm{K}$, et al. : IDH1 mutations in diffusely infiltrating astrocytomas: grade specificity, association with protein expression, and clinical relevance. Am J Clin Pathol 138 : 177-184, 2012

25. Thotakura M, Tirumalasetti N, Krishna R : Role of Ki-67 labeling index as an adjunct to the histopathological diagnosis and grading of astrocytomas. J Cancer Res Ther $10: 641-645,2014$

26. Turkalp Z, Karamchandani J, Das S : IDH mutation in glioma: new insights and promises for the future. JAMA Neurol 71 : 1319-1325, 2014

27. Wang HY, Tang K, Liang TY, Zhang WZ, Li JY, Wang W, et al. : The comparison of clinical and biological characteristics between IDH1 and IDH2 mutations in gliomas. J Exp Clin Cancer Res 35 : 86, 2016

28. Wang Z, Zhang C, Liu X, Wang Z, Sun L, Li G, et al. : Molecular and clinical characterization of PD-L1 expression at transcriptional level via 976 samples of brain glioma. Oncoimmunology 5 : e1196310, 2016

29. Xue S, Hu M, Li P, Ma J, Xie L, Teng F, et al. : Relationship between expression of PD-L1 and tumor angiogenesis, proliferation, and invasion in glioma. Oncotarget 8 : 49702-49712, 2017

30. Zeng A, Hu Q, Liu Y, Wang Z, Cui X, Li R, et al. : IDH1/2 mutation status combined with $\mathrm{Ki}-67$ labeling index defines distinct prognostic groups in glioma. Oncotarget 6 : 30232-30238, 2015 